\title{
Essais
}

ESSAIS

Revue interdisciplinaire d'Humanités

5 | 2014

Médias et élites

\section{Communication Humain/Machine en langue naturelle écrite : quel partage du sens?}

Maria-Caterina Manes-Gallo

\section{OpenEdition}

1 Journals

Édition électronique

URL : http://journals.openedition.org/essais/8267

DOI : $10.4000 /$ essais. 8267

ISSN : 2276-0970

Éditeur

École doctorale Montaigne Humanités

Édition imprimée

Date de publication : 15 juillet 2014

Pagination : 126-136

ISBN : 978-2-9544269-3-8

ISSN : 2417-4211

Référence électronique

Maria-Caterina Manes-Gallo, «Communication Humain/Machine en langue naturelle écrite : quel

partage du sens? », Essais [En ligne], 5 | 2014, mis en ligne le 13 avril 2021, consulté le 15 avril 2021.

URL : http://journals.openedition.org/essais/8267 ; DOI : https://doi.org/10.4000/essais.8267 


\section{Communication Humain/ Machine en langue naturelle écrite : quel partage du sens?}

\section{Maria-Caterina Manes-Gallo}

La question liminaire posée dans la présente contribution concerne les retombées en sciences humaines et sociales des travaux de représentation des connaissances, finalisés à la conception d'interfaces appliquées à la recherche d'information en texte intégral. La problématique sous-jacente est celle de la communication entre un humain et une machine en langue naturelle écrite. Ce qui inscrit ces travaux dans le domaine du Traitement automatique de la langue naturelle.

Dans l'exposé qui suit, il s'agira d'illustrer comment les contraintes, qui pèsent sur la modélisation des connaissances linguistiques pour le processus de reconnaissance automatique, peuvent devenir une heuristique féconde pour approfondir la dimension foncièrement polysémique de toute marque langagière. Notamment les constructions prédicatives.

\section{Introduction}

L'objet de mon exposé concerne la communication Humain/Machine $(\mathrm{H} / \mathrm{M})$ en langue naturelle écrite (LNE). Il s'inscrit dans une problématique théorique finalisée à la conception de dispositifs d'interface, appliqués à la recherche d'information (en particulier en texte intégral). La fonction d'intermédiaire de ces dispositifs pose en premier plan leur capacité, à terme, «d'avoir l'air de comprendre » un discours en langue naturelle écrite (texte ou tour de parole dans un dialogue avec l'utilisateur humain). Il s'agit de prendre en compte la dissymétrie cognitive et communicationnelle des deux partenaires face à l'instrument langagier qui contribue à la mise en forme signifiante de l'information échangée. D'où l'inscription de cette problématique dans un contexte de Traitement Automatique de la Langue Naturelle Écrite (TALNE).

À partir d'une approche interdisciplinaire qui se situe au carrefour des sciences de l'information et de la communication (SIC) et des sciences du langage (SdL), l'objectif est de montrer comment les enjeux théoriques et méthodologiques 
soulevés par la conception d'interfaces plus " anthropocentrées » alimente la réflexion sur la question du sens langagier en sciences humaines.

La fonction d'intermédiaire du dispositif d'interface est d'extraire le sens de la requête d'un utilisateur, afin de l'apparier avec un fragment de texte pertinent et, si besoin est, de relancer l'utilisateur afin qu'il précise sa requête. Côté interface, ces deux phases de l'interaction avec un humain, renvoient à deux catégories différentes de processus de traitement des séquences langagières. Notamment, des processus de reconnaissance du sens et des processus de génération de séquences douées de sens.

Dans le cadre des processus de reconnaissance, j’approfondirai un aspect de la représentation des connaissances linguistiques mobilisées dans le traitement sémantique des énoncés élémentaires, i.e. des séquences organisées autour d'un élément prédicatif et hors connecteurs. L'impossibilité de représenter en un seul système les connaissances encyclopédiques, sous-jacentes au processus d'attribution du sens chez l'humain, contribue à projeter en premier plan l'importance des modalités énonciatives par lesquelles la mise en fonctionnement de la langue dans un discours écrit contribue à l'émergence et au " partage du sens ». Le modèle de sémantique énonciative qui sera exposé vise à rendre compte de la fonction des constructions prédicatives par rapport à la progression d'un discours. L'illustration d'un exemple de son application permettra de montrer comment les contraintes méthodologiques qui pèsent sur le travail théorique, sous-jacent à "l'instrumentation " du sens langagier, contribuent à l'approfondissement des relations entre la valence re/présentationnelle des productions langagières et leur fonction communicationnelle.

\section{La communication $\mathrm{H} / \mathrm{M}$ en Sciences de l'information et de la communication}

Historiquement, la communication H/M n'apparaît pas, dès sa fondation dans les années 1970, comme un champ d'investigation spécifique aux SIC. Problématique transversale, commune à plusieurs disciplines (notamment l'informatique, l'ergonomie, la psychologie cognitive...), la communication $\mathrm{H} / \mathrm{M}$ est étroitement liée à la naissance de l'Intelligence Artificielle d'abord et à celle des Sciences Cognitives ensuite.

Avec l'automatisation du traitement du document et de l'information vers la fin des années 1950 et ensuite l'avènement d'Internet vers la fin des années 1990, les SIC se sont progressivement intéressées à l'étude des interactions entre un humain et une machine et entre des humains par l'intermédiaire de dispositifs informatiques. Les travaux en communication $\mathrm{H} / \mathrm{M}$ prennent en compte ces processus de médiation à partir de deux perspectives différentes, en focalisant l'attention soit sur l'aspect communicationnel soit sur l'aspect informationnel de l'interaction entre un humain et une machine. 
Le premier courant, plus orienté vers l'étude des processus médiatiques, s'intéresse à l'articulation entre technologies et société, à partir d'un double questionnement : comment saisir l'action de la technique dans la société ? Et comment prendre en compte l'action du contexte socio-économique sur le développement des inventions techniques?

Avec l'avènement d'Internet et l'évolution des techniques de gestion électronique des documents (e.g. apparition de systèmes de recherche d'informations textuelles), la recherche documentaire est devenue le quotidien de tous, indépendamment du niveau de familiarité avec l'outil informatique et de la visée de l'activité de s'informer (e.g. travail, loisirs...). D'où la nécessité de prendre en compte et de répondre à la question des décalages existants entre l'activité de sinformer telle qu'elle est mise en œuvre par un humain et les fonctionnalités offertes par l'intermédiation informatique, notamment du dispositif d'interface (e.g. format des requêtes, indexation automatique du contenu de documents...).

C'est dans ce second courant que s'inscrivent les problèmes théoriques et méthodologiques soulevés par la conception de dispositifs d'interface capables, à terme, "d'avoir l'air de comprendre " le sens d'une information inscrite sous forme langagière. Des relations privilégiées avec la psychologie cognitive et les SdL viennent alimenter l'interdisciplinarité des SIC.

\section{La recherche d'information en texte intégral : petit détour}

La recherche d'information (RI) est une activité dont la finalité est de mettre en regard une information et un utilisateur. Elle est généralement finalisée à un objectif (professionnel ou de loisirs) qui transcende l'activité de recherche ellemême. Le rôle d'intermédiaire de l'interface est de mettre en correspondance la logique de fonctionnement du système de gestion des documents (e.g. descripteurs associés aux documents, algorithmes de gestion) et la logique d'utilisation de l'humain (e.g. variétés des tâches, complexité des interrogations, niveau de connaissance des fonctionnalités du système...) ; ceci afin de combler (idéalement) le décalage entre les deux logiques.

L'interface a pour fonction d'extraire le sens de la requête de l'utilisateur et de l'apparier avec des documents (recherche documentaire) ou des fragments de documents (recherche en texte intégral), à partir de l'indexation de leur contenu. L'enjeu étant de fournir à l'utilisateur des unités d'information à la fois utiles (taux de rappel élevé vs. silence) et utilisables (taux de précision vs. bruit) par rapport aux attentes formulées dans sa requête. Dans la recherche en texte intégral, l'enjeu est de réussir à indexer de façon automatique son contenu, en exploitant les caractéristiques linguistiques de son inscription. Linterrogation (e.g. par mots clés, thèmes ou auteur...) pourra se faire en exploitant les différentes parties du document, grâce à une procédure de localisation automatique des différents 
thèmes occurrents (e.g. dans un Manuel technique : le fonctionnement du dispositif vs. les procédures de maintenance). La définition de cette procédure s'appuie d'un côté sur la segmentation du contenu en unités d'information distinctes et sémantiquement autonomes et de l'autre sur l'identification des différents thèmes traités, à partir des caractéristiques de leur " mise en mots ». Le problème de la reconnaissance automatique des modalités de mise en fonctionnement de la langue qui configurent le contenu d'un document, inscrit la RI en texte intégral dans le domaine du TALNE. Un des objectifs devient la définition de système de représentation des connaissances à fort ancrage langagier.

\section{Le «partage du sens » comme problème}

La conception des dispositifs d'interface se heurte à plusieurs difficultés qui constituent l'objet des recherches interdisciplinaires en communication H/M (Calvary, 2002). La première difficulté est due à l'indirection utilisateursystème via le concepteur. Le concepteur n'est pas un utilisateur et l'utilisateur n'est pas le concepteur. D'où la nécessité de définir préalablement des modèles comportementaux plus ou moins prototypiques (e.g. les interfaces réactives des bornes automatiques de ventes de billets SNCF).

La seconde difficulté dépend de l'activité de RI qui n'est pas une activité balisée, mais une activité de résolution de problèmes mal structurés (Dinet, Rouet, 2002). L'objectif informationnel de l'utilisateur, n'étant identifiable qu'à partir d'un manque de connaissance, il est rarement formulé de façon explicite. D'où la difficulté de définir a priori quel est l'objectif final à réaliser, i.e. quelle est l'information pertinente en regard des attentes de l'utilisateur. Ce qui est à l'origine de la troisième difficulté.

Pour identifier quelle peut être l'information recherchée par l'utilisateur la seule possibilité est d'inférer cette dernière à partir de la formulation de ses requêtes. Il s'agit de doter l'interface de connaissances langagières pour pallier le décalage entre les deux partenaires. Notamment, par rapport à l'opaque polysémie du mode langue naturelle, en tant que moyen de mise en forme de l'information. D'autant plus que l'humain et le dispositif ne traitent pas la même entité " information ".

Comme le souligne Edgar Morin le terme « information » renvoie à une notion nucléaire, dont il est difficile de se passer, mais qui demeure difficile à cerner à cause de l'extraordinaire migration disciplinaire dont elle a été l'objet. Le terme "information ", loin de renvoyer à un concept élucidant et/ ou élucidé, constitue le point de départ d'une réflexion qui doit en concevoir le caractère radical et poly-dimensionnel (Morin, 2005). C'est ce caractère poly-dimensionnel du terme "information " que l'instrumentation du sens oblige à prendre en compte. 
L'intérêt, de la distinction formulée par le philosophe Raymond Ruyer entre information objective et information subjective (Ruyer, 1954) est d'approfondir comment le référent du terme "information " se transforme lorsqu'on passe du dispositif informatique à l'être humain. D'une entité quantifiable et observable, traitée comme une donnée, ou une structure, l'information devient une valeur, qui dépend du sens qu'elle acquiert pour les partenaires humains qui l'échangent. Le sens ne fait pas l'objet d'un transfert comme une donnée : il est reconnu, reconstruit ou généré, par des agents cognitifs, à partir d'une symbolisation qui le rend communicable, c'est-à-dire partageable. Ainsi l'information subjective ne peut pas être considérée, à l'instar de l'information objective, comme une donnée mais comme un construit, i.e. le résultat signifiant d'un processus de symbolisation (Floridi, 2005).

Cette opposition entre information objective et information subjective sous tend la dissymétrie cognitive caractéristique de la communication H/M en langue naturelle. Le dispositif d'interface traite de l'information (des données), en manipulant des séquences de caractères qui pour lui n'ont aucun sens et dont la signification est calculée à partir des données de connaissance qui ont été représentées dans le programme par le concepteur du système. En revanche, la familiarité de l'humain avec le mode langue naturelle va de pair avec sa capacité de reconstruire à partir de la combinatoire de formes langagières un sens implicite qui configure l'information subjective comme un construit signifiant.

Dans ce cadre, pour la communication H/M l'enjeu est de doter le dispositif d'un système de représentation des connaissances à fort ancrage langagier qui lui permette de "partager du sens » avec l'utilisateur.

\section{Le rôle de la représentation des connaissances}

Historiquement, la représentation des connaissances a constitué le socle dur de l'Intelligence Artificielle. Depuis l'article inaugural de John Mc Carthy et Patrick J. Hayes (1969), on distingue deux aspects conceptuellement distincts dans la conception des programmes informatiques : l'aspect épistémologique et l'aspect heuristique. Le premier aspect concerne la modalité à travers laquelle on modélise le problème que le système doit résoudre. Par exemple, pour que le système ait l'air de comprendre le message de l'utilisateur il faudra lui fournir des connaissances sur les différents niveaux conceptuels de fonctionnement des formes langagières (morphologique, syntaxique, sémantique et pragmatique). Ces connaissances détermineront l'exhaustivité des calculs effectués par l'interface, afin d'extraire automatiquement le sens d'une ou plusieurs séquences langagières. L'aspect heuristique concerne en revanche la définition d'algorithmes efficaces pour la recherche automatique de la solution d'un problème. Par exemple, les procédures pour éliminer les 
solutions parasites de formes linguistiques homographes (e.g. elle lit un livre vs. elle est assise sur le lit). Ces deux aspects bien que distincts sont étroitement liés : la complexité de l'aspect épistémologique rejaillit sur l'aspect heuristique. La collaboration entre informatique et sciences humaines concerne surtout l'aspect épistémologique de la représentation des connaissances.

Si visualisée de l'intérieur, une interface en langue naturelle peut être décrite intuitivement comme un système de transformation des représentations du sens véhiculé par des séquences langagières aux différents niveaux d'analyse, définis par les linguistes. Étant donné un énoncé en entrée, l'objectif de l'analyse syntaxique, par exemple, est de regrouper en syntagmes, selon leur catégorie grammaticale d'appartenance, les formes (groupe de morphèmes) qui constituent l'énoncé.

Au niveau sémantique, en revanche, il s'agira de déterminer qu'elle est l'interprétation associable aux constituants dégagés par l'analyse syntaxique et quelles sont leurs relations au sein de la séquence analysée. Le traitement en sémantique est plus complexe parce qu'il peut être mis en œuvre en référence à deux niveaux de granularité différents. Notamment, au niveau de chaque lexème (sémantique lexicale) occurrent dans un syntagme ou bien au niveau des relations sémantiques qu'entretiennent les syntagmes occurrents dans une séquence (e.g. un énoncé simple). Dans le modèle exposé plus bas ces relations sont analysées à partir de la direction de prédication des constructions prédicatives.

\section{Exposé synoptique du modèle de sémantique énonciative}

Le modèle vise à rendre compte de la fonction des constructions prédicatives (verbe + les actants qui l'encadrent) par rapport à la progression d'un discours. Il a été défini comme une composante d'un système de représentation des connaissances finalisé au traitement pragmatique. Ce dernier doit permette de reconnaître, à partir des données textuelles, quels sont les objets de discours, ce dont on parle dans un texte ou une requête, et l'évolution des propriétés qui leur sont attribuées : comment on en parle, au fur et à mesure que progresse le discours. Les représentations construites au niveau pragmatique constituent une mémoire discursive qui détermine au niveau opérationnel l'exhaustivité des calculs effectués par l'interface pour trouver des appariements satisfaisants entre le sens extrait d'une requête et les portions de texte indexées (Rouault, Manes-Gallo, 2003).

Dans le cadre d'un énoncé simple, l'ancrage langagier qui actualise l'évolution des propriétés des objets du discours est porté par les constructions prédicatives. Le type de procès lexical, référent du prédicat, détermine sa direction de prédication. La direction de prédication induit l'attribution d'une nouvelle propriété respectivement soit à l'actant de gauche (sujet grammatical), soit à l'actant de droite, (argument régi). La reconnaissance au niveau pragmatique 
de l'évolution des propriétés des objets de discours est donc articulée à la direction de prédication des constructions prédicatives, déterminée par leur type de procès référent. Cependant ce dernier peut subir des glissements selon le co-texte d'occurrence du verbe ; d'où la nécessité de définir des données de connaissances qui permettent au traitement sémantique de reconnaître automatiquement cette dimension de la polysémie des prédicats.

Très synthétiquement, la direction de prédication des constructions prédicatives est définie en référence : (a) aux types de procès lexical auxquels elles renvoient et (b) aux facteurs co-textuels qui transforment leur type de procès lexical, en induisant un glissement vers un autre type de procès. Le schéma métalinguistique qui permet de représenter de façon homogène les transformations du type de procès lexical est une adaptation de la notion de lexis élaboré par Antoine Culioli (1990).

Pour le point (a), le type de procès lexical auquel renvoie un verbe est déterminé en référence au schéma syntaxique de base $\mathrm{C} 0$ - $\mathrm{V}$ - $\mathrm{C} 1$ pour les verbes transitifs et $\mathrm{C} 0-\mathrm{V}$ pour les verbes intransitifs ${ }^{1}$. Une prédication peut renvoyer alternativement à une situation statique (état) ou à une situation dynamique (processus). Cette dernière peut évoquer soit l'absence d'accomplissement, soit un accomplissement (Gallo et Rouault, 1992). Dans le dernier cas, on parle de processus résultatif: il induit l'attribution implicite d'une nouvelle propriété à l'actant de droite (il prend un $R V$ chez le médecin! le RV est pris). En revanche, lorsque le processus référent n'évoque pas un accomplissement, on parle de processus non résultatif (e.g. le vernis prend lentement). Par hypothèse, on considère que dans ce dernier cas et dans le cas de l'état (e.g. on lui connaît des mérites) les constructions prédicatives affectent plutôt les propriétés de l'actant de gauche, ou les relations que ce dernier entretient avec les autres objets du discours. Ce dernier point sera illustré plus bas, à partir de l'analyse de l'énoncé :

L'union et les états membres prennent des mesures pour accroître la fourniture du transport ferroviaire.

D'après le modèle, les transformations du type de procès lexical (Cf. point (b) plus haut) peuvent être induites alternativement ou simultanément par les facteurs co-textuels suivants :

b-1) les schémas syntaxiques alternatifs associables au même lexème verbal : l'emploi intransitif d'un processus résultatif induit l'attribution d'une propriété à l'actant de gauche ;

b-2) les caractéristiques sémantiques des arguments qui occupent les différentes places syntaxiques à gauche et à droite du verbe. C'est le cas de

1 Les tests métalinguistiques adoptés pour identifier le type de procès lexical sont : la transformation à la forme progressive et l'autonomie de la construction passive. Par exemple : Jean construit une maison! la maison est construite (Fuchs, Léonard 1979). 
l'instrument: les traits sémantiques du sujet grammatical ne permettent plus d'évoquer une situation dynamique (e.g. *le câble est en train de connecter le dispositif au serveur). C'est aussi le cas de la signification inchoative de certains processus résultatifs induite par l'imperméabilité du complément d'objet par rapport à la visée portée par le verbe (e.g. prendre un studio en ville vs. prendre la première rue à gauche) (Manes-Gallo, 2007) ;

b-3) les opérations d'énonciation qui accompagnent l'actualisation de la construction prédicative. Notamment, les déterminants associés aux arguments qui encadrent le verbe, les modalités, les temps et l'aspect, les compléments circonstanciels associés...

L'exemple décrit dans le paragraphe suivant a pour objectif d'illustrer ces effets du cotexte dans le cas du verbe prendre.

\section{Un exemple d'application}

L'objectif dans ce paragraphe est d'illustrer les problèmes sous-jacents à la reconnaissance automatique de la fonction de constructions prédicatives organisées autour du verbe prendre, par rapport à la progression du discours. Il s'agit de caractériser la variation du type de procès lexical auquel renvoie ce verbe dans son emploi transitif, à partir d'un exemple tiré du projet de Constitution européenne, soumis au référendum de 2005 (Duhamel, 2005). L'énoncé à l'étude est : l'union et les états membres prennent des mesures pour accroître la fourniture du transport ferroviaire.

Comme indiqué précédemment (cf. $\$$ IV), l'interface est un système de transformation des représentations du sens d'une séquence langagière. Dans ce qui suit on part des résultats du traitement syntaxique qui est : a') le schéma SN1 V1 SN2 <préposition> Vinf2 SN3.

Un module de passage entre traitement syntaxique et traitement sémantique va étiqueter les constituants syntaxiques selon leur rang. Le schéma (a') est transformé dans le schéma : C0 V C1 C5pour (0 V’ C1'). Cette étape permet de distinguer quels sont les compléments régis $(\mathrm{C} 1$ pour $\mathrm{V}$ et $\mathrm{Cl}^{\prime}$ pour $\mathrm{V}^{\prime}$ ) et les compléments circonstants (C5). Elle est indispensable pour déterminer au niveau sémantique quel est le RÔLE des arguments qui encadrent le prédicat, et leurs relations, selon le type de procès auquel il renvoie.

D'après le modèle, le traitement sémantique va générer plusieurs lexis de la séquence. Ces schémas servent à reconnaître le type de procès lexical du verbe, à partir de l'activation d'un dictionnaire des verbes, et à déterminer si le co-texte (i.e. rang des compléments, traits sémantiques des arguments, opérations d'énonciation) exerce ou pas un effet sur la direction de prédication (i.e. transforme ou pas le type de procès lexical). Dans l'exemple, il s'agit d'établir si les éléments du co-texte altèrent le fonctionnement lexical de prendre, 
qui coïncide avec un processus résultatif. D’après le modèle, la stabilité de ce dernier est conservée par deux éléments : les traits sémantiques du C0 et la perméabilité du C1 par rapport à la visée du processus (cf. \$5).

Dans l'exemple à l'étude, le $\mathrm{C} 0$ demeure une causalité extrinsèque qui joue le RÔLE d'agent par rapport au déclenchement et à l'accomplissement du processus prendre. Cependant le $\mathrm{C} 1$ joue un double rôle : il est à la fois l'objet visé par le processus prendre et l'instrument, ou la causalité intrinsèque, qui permet la réalisation de l'objectif explicité par le complément C5, introduit par la préposition pour. Il constitue le C0 implicite du V2 accroître. L'énoncé de départ peut en effet être paraphrasé par : l'union et les états membre prennent les mesures qui accroissent la fourniture du transport ferroviaire. Le schéma suivant permet de mieux visualiser la situation :

L'union et les états membres prennent des mesures pour accroître la fourniture du transport ferroviaire.

Mesures.

"Causalité intrinsèque ".

Cette double fonction du C1 contribue à faire glisser prendre dans la catégorie des processus non résultatifs. Le prédicat n'attribue pas une nouvelle propriété au C1. Via le complément C5 introduit par la préposition pour, le $\mathrm{C} 1$ contribue à établir une nouvelle relation entre l'institution (i.e. l'union qui apparait comme sujet grammatical de prendre) et un de ses domaines de compétence (i.e. accroître la fourniture du transport ferroviaire), (Manes-Gallo, Rouault, 2010).

\section{Quelles retombées en sciences humaines et sociales?}

Dans le cadre de la (RI) en texte plein, les connaissances linguistiques représentées dans l'interface sont à l'origine du degré d'efficacité du processus d'appariement entre le sens de la requête de l'utilisateur et la pertinence des portions de texte pouvant combler le besoin d'information de ce dernier. Un des objectifs est de pallier la dissymétrie, à la fois cognitive et communicationnelle entre les deux partenaires, par rapport à la reconnaissance du sens de l'information échangée.

Le travail sur la dimension épistémologique de la représentation des connaissances oblige à définir en quoi consiste la connaissance partagée sur la LN, en évitant le recours circulaire aux connaissances encyclopédiques qui en permettent l'appréhension par un humain [Manes-Gallo, 2007 (a)]. C'est dans le creuset de cette indépendance du sens construit par le discours, que les contraintes liées à l'« instrumentation » du sens deviennent une méthodologie précieuse pour approfondir la dimension signifiante de tout information inscrite sous forme langagière. 
Le travail théorique, orienté vers une plus grande explicitation de l'opaque polysémie générée par toute mise en fonctionnement de la langue naturelle dans un discours. L'intérêt de l'entreprise est d'inviter à réfléchir sur "l'évidence " du fonctionnement de la langue naturelle comme instrument de communication et comme vecteur de la cognition. Dans le travail présenté cette " évidence " concerne la variabilité des effets de sens que les différentes utilisations d'un même prédicat permettent d'évoquer. Ce sont ces « effets de sens " inédits et provisoires qui pour l'humain configurent l'information, non pas comme une donnée, mais toujours comme un "construit ", résultant de la progression d'un discours.

Maria-Caterina Manes-Gallo EA 4195 TELEM

Université Bordeaux Montaigne Maria-Caterina.Manes-Gallo@u-bordeaux-montaigne.fr

\section{Mots clés}

Recherche documentaire, représentation des connaissances, schéma sémantique, prédication verbale.

\section{RÉFÉRENCES BIBLIOGRAPHIQUES}

Calvary G., Ingénierie de l'interaction homme-machine : rétrospective et perspectives, in Céline Paganelli (éd.), Interaction Homme-Machine et recherche d'information, Paris, Hermès-Lavoisier, 2002.

Culioli A., Pour une linguistique de l'énonciation, Paris, Ophrys, 1990.

Dinet J., Rouet J.-F., La recherche documentaire informatisée : processus cognitifs, facteurs de difficultés et dimension de l'expertise, in Céline Paganelli (éd.), Interaction HommeMachine et recherche d'information, Paris, Hermès-Lavoisier, 2002.

Duhamel O., La constitution européenne : texte définitif présenté par Olivier Duhamel, Paris, Armand Colin, 2005.

Floridi L., Semantic conceptions of information, Stanford Encyclopedia of Philosophy, 2005, http://plato.stanford.edu/entries/information-semantic/.

Fuchs C., Léonard, A.-M., Vers une théorie des aspects. Paris, Mouton, 1979.

Manes-Gallo M.-C., Rouault J., Schémas d'action et types de procès. Intellectica, 1, 1992, p. 105-127.

Manes-Gallo M.-C., La prédication verbale entre progression du discours et progression dans l'espace, in Jean-Marie Barbéris et Maria-Caterina Manes-Gallo (éd.), Parcours dans la ville: La description d'itinéraires piétons, Paris, Éditions de l'Harmattan, 2007 (a), p. 211-230.

Manes-Gallo M.-C., "Sur les outils informatiques pour les études empiriques inter-disciplinaires : problèmes de conception et nouvelles pratiques ", in Jean-Marie Barbéris et Maria-Caterina Manes-Gallo (éd.) Revue Interaction Homme/Machine, vol. 8, n 2, 2007 (b), http://www.europia.org/RIHM/. 
Manes-Gallo M.-C., Rouault J., Variations syntactico-sémantiques sur la polysémie du verbe "prendre ", in Camino Alverez Castro, Flor Maria Bango de la Campa et Maria Luisa Donaire (éd.), Liens linguistiques : Études sur la combinatoire et la hiérarchie des constituants, Bern, Peter Lang, 2010, p. 427-443.

Mc Carthy J., Hayes P.-J., Some philosophical problems from the standpoint of artificial intelligence, in David Michie D. et Bernard Meltzer (éd.), Machine Intelligence, $\mathrm{n}^{\circ} 4$, Edinburgh, Edinburgh University Press, 1969, p. 600-610.

Morin E., Introduction à la pensée complexe, Pairs, Seuil, 2005.

Rouault J., Manes-Gallo M.-C., Intelligence Linguistique : Le couple sémantique-pragmatique et le calcul du sens des énoncés élémentaires, Paris, Hermès-Science, 2003.

Ruyer R., La cybernétique et l'origine de l'information, Paris, Flammarion, 1954. 